\title{
Recurrent Paget Disease of the Breast
}

National Cancer Institute

\section{Source}

National Cancer Institute. Recurrent Paget Disease of the Breast. NCI Thesaurus. Code C153588.

The reemergence of Paget disease of the breast after a period of remission. 